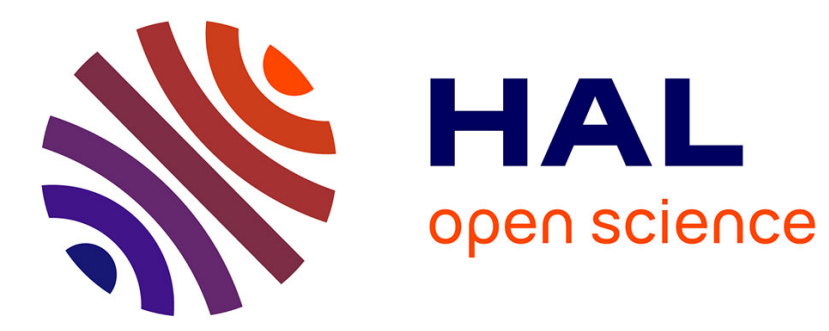

\title{
Physical mesomechanical criteria of plastic deformation and fracture
}

\author{
S. Yoshida, R.L. Rourks, T. Mita, K. Ichinose
}

\section{To cite this version:}

S. Yoshida, R.L. Rourks, T. Mita, K. Ichinose. Physical mesomechanical criteria of plastic deformation and fracture. Physical Mesomechanics, 2009, 12 (5-6), pp.249-253. hal-01092733

\section{HAL Id: hal-01092733 \\ https://hal.science/hal-01092733}

Submitted on 9 Dec 2014

HAL is a multi-disciplinary open access archive for the deposit and dissemination of scientific research documents, whether they are published or not. The documents may come from teaching and research institutions in France or abroad, or from public or private research centers.
L'archive ouverte pluridisciplinaire HAL, est destinée au dépôt et à la diffusion de documents scientifiques de niveau recherche, publiés ou non, émanant des établissements d'enseignement et de recherche français ou étrangers, des laboratoires publics ou privés. 


\title{
Physical mesomechanical criteria of plastic deformation and fracture
}

\author{
S. Yoshida*, R.L. Rourks, T. Mita ${ }^{1}$ and K. Ichinose ${ }^{1}$ \\ Southeastern Louisiana University, SLU 10878, LA 70402, USA \\ ${ }^{1}$ Tokyo Denki University, Tokyo, 101-8457, Japan
}

\begin{abstract}
A method is proposed to identify the near crack-tip region in a deforming object as the plastic zone and to diagnose its status as to whether or not the crack is about to open. The in-plane displacement field is visualized as a two-dimensional, full-field optical interferometric fringe pattern, and the diagnosis is made based on the plastic deformation and critical fracture criteria derived from a recent theory of deformation and fracture called physical mesomechanics. The proposed method is demonstrated for tensile experiments conducted for tin and steel specimens.

Keywords:
\end{abstract}

\section{Introduction}

The transition from plastic deformation to fracture in solid-state materials is a complicated process, and its physics has not been fully understood. Current theory based on conventional fracture mechanics [1] is in most part phenomenological, relying on the elastic theory to formulate the highly inelastic fracturing process. The boundary of the near crack-tip plastic zone is estimated from a mathematically modeled stress distribution, and a yield stress obtained in a separate experiment. The condition of unstable crack propagation is evaluated from the energy balance between the elastic energy stored in the material and the energy release associated with the generation of free surfaces. It is not necessarily connected with an equation of motion that describes the dynamics of the fracturing zone. This situation imposes limitations on accurate prediction of fracture in various engineering applications. More physically solid, universal approach is desirable.

In this work, we present a universal approach to analyze the deformation field of ductile materials in the prefracture stage. Using tensile experiments conducted for tin and steel specimens as an example, we demonstrate that it is possible to identify the near crack-tip region as the plastic zone and to diagnose its status as to whether or not the crack is about to open. The displacement field of the speci-

\footnotetext{
* Corresponding author

Prof. Sanichiro Yoshida, e-mail: syoshida@selu.edu
}

men is visualized as a full-filed image with the use of an optical interferometric technique known as the electric speckle-pattern interferometry [2], and the plastic zone is identified from the interferometric fringe pattern. The diagnosis is based on the plastic deformation and critical fracture criteria derived from physical mesomechanics [3], a recent universal theory of deformation and fracture. The universality of physical mesomechanics results from the fact that it is founded on the gauge invariance, one of the most fundamental principles in physics. Because of this universality, physical mesomechanics is capable of describing all the stages of deformation comprehensively. The plastic deformation and critical fracture criteria are based on this foundation.

\section{Physical mesomechanics and field equation}

The gauge theoretical formalism of physical mesomechanics can be conveniently understood as an extension of the global symmetry in elasticity to local symmetry in plasticity [4]. In the theory of elasticity [5], the distortion tensor components, known as the normal strain, shear strain and rotation, are constants. The fact that the rotation tensor component is constant indicates that the material rotates as a rigid body. In physical mesomechanics, the plasticity is characterized as the situation where the distortion tensor components are coordinate dependent $[4,6]$. Intuitively, this can be understood as follows. Imagine that a defect is generated inside a deforming material. Naturally, the blocks 
around the defect will have the freedom of rotating differently; the rotation becomes coordinate dependent, not rigidbody rotation. Since the distortion tensor components contain spatial derivatives, this requires the replacement of the usual derivatives with covariant derivatives, or the introduction of a gauge term. With the application of the Lagrangian formalism to the gauge field, this leads to the following Maxwell type field equations [6]. The general solution to this field equation is a decaying transverse wave [7] of the translational and rotational displacement:

$$
\begin{aligned}
& \nabla \cdot \mathbf{v}=j_{0}, \\
& \nabla \times \boldsymbol{\omega}=-\varepsilon \mu \frac{\partial \mathbf{v}}{\partial t}-\mathbf{j},
\end{aligned}
$$

where $\mathbf{v}$ and $\boldsymbol{\omega}$ are the rate of the translational displacement and the rotational displacement (angle from the rotational equilibrium), respectively, $\varepsilon$ is the density of the material, and $1 / \mu$ is the shear modulus [8], $j_{0}$ and $\mathbf{j}$ are the currents of symmetry (the time and space components, respectively) [6]. Putting $j_{0}=\rho / \varepsilon$ in Eq. (1) and taking divergence of Eq. (2), we can derive the equation of continuity in the following form:

$$
\frac{\partial \rho}{\partial t}=-\frac{1}{\mu} \nabla \cdot \mathbf{j} .
$$

Here, $\rho=\varepsilon \nabla \cdot \mathbf{v}$ can be interpreted as the divergence of momentum density associated with strain concentration $\nabla \cdot \mathbf{v}$. Equation (3) allows us to put the current $\mathbf{j}$ in the form of the product of the charge density $\rho$ and its drift velocity $W_{\mathrm{d}}$ as

$$
\frac{\mathbf{j}}{\mu}=\rho \mathbf{W}_{\mathrm{d}}=(\varepsilon \nabla \cdot \mathbf{v}) \mathbf{W}_{\mathrm{d}} \text {. }
$$

Recent analysis [8] reveals that the above field equation reduces to the following equation of motion that describes the dynamics of an infinitesimal volume in a plastically deforming object:

$$
\varepsilon \frac{\partial \mathbf{v}}{\partial t}=-\frac{1}{\mu}(\nabla \times \boldsymbol{\omega})-\frac{1}{\mu} \mathbf{j} .
$$

The left-hand side of Eq. (5) is the change in momentum of the unit volume. The right-hand side represents the external forces acting on the unit volume where the first term represents the recovery force in the plastic regime, and the second term represents the longitudinal force [8]. In the elastic regime the longitudinal force is proportional to the displacement, and hence is a recovery force. In the plastic regime it is proportional to the velocity, and hence is an energy dissipative force. Equation (3) can be interpreted as that the change in the strain concentration is caused only by the differential longitudinal force.

\section{Mesomechanical criteria of deformational stages}

The above definition of plasticity based on the local symmetry leads to the criteria that deformation is (a) elastic if the first order spatial derivatives of displacement are coordinate independent, and (b) plastic if they are coordinate dependent. Further analysis leads to a criterion that describes that the material is about to fracture $[6,9]$. This criterion, called the critical fracture criterion, is given as below:

$$
\begin{aligned}
& \nabla \times \boldsymbol{\omega}=0, \\
& \mathbf{j} \neq 0 .
\end{aligned}
$$

With the help of Eq. (5), Eq. (6) can be understood as the situation where the material loses its capability of exerting the plastic recovery force, while the field energy is dissipated via the longitudinal plastic force as represented by Eq. (7) [8]. In this situation, as the deformation progresses under the influence of tensile loading, for instance, the material keeps stretching until it fractures.

\section{Experimental arrangement}

Detailed description of the electronic speckle-pattern interferometry can be found elsewhere [2]. In short, a typical electronic speckle-pattern interferometry setup consists of two laser beams reflected on an object surface and recombined on the image plane of an electronic imaging device such as a charge-coupled device. On the image plane, the speckle field associated with the first optical interferometric path is superposed with the speckle field associated with the second interferometric path. Consequently, at each point on the image plane, the total intensity contains the relative phase of the speckles associated with the two paths:

$$
I=A_{1}^{2}+A_{2}^{2}+2 A_{1} A_{2} \cos \left(\theta_{1}-\theta_{2}\right),
$$

where $A_{1}, A_{2}$ and $\theta_{1}, \theta_{2}$ represent the amplitude and phase of the speckles corresponding to the two paths. The image frame represented by Eq. (8) is called the specklegram. If the object surface displaces causing a relative phase changes from $\theta_{1}-\theta_{2}$ to $\theta_{1}-\theta_{2}+\phi$, the total intensity becomes

$$
I^{\prime}=A_{1}^{2}+A_{2}^{2}+2 A_{1} A_{2} \cos \left(\theta_{1}-\theta_{2}+\phi\right) \text {. }
$$

Figure 1 illustrates the experimental arrangement used in this study. Two in-plane sensitive electronic speckle-pattern interferometry setups are arranged on the front and rear sides of the specimen attached to a tensile machine. The

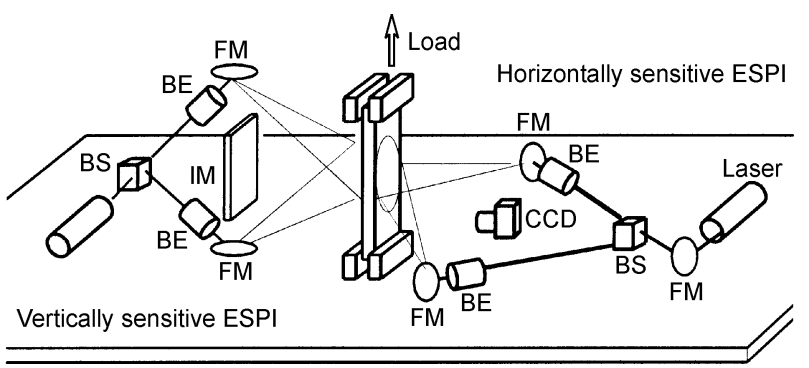

Fig. 1. Experimental setup: ESPI - electric speckle-pattern interferometry, FM - folding mirror, BS - beam splitter, BE - beam expander, IM - imaging mirror. Multiple imaging mirrors were used to make the object distances for the front and rear surfaces of the specimen the same, but only one is shown in this figure to avoid complexity 


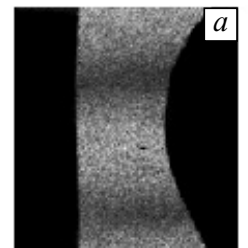

$41 \mathrm{H}$

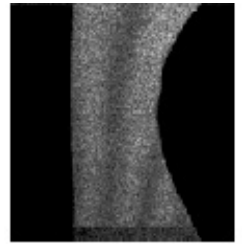

$42 \mathrm{~V}$

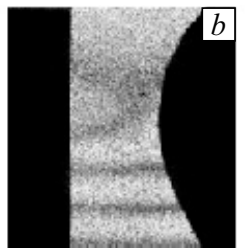

$402 \mathrm{H}$

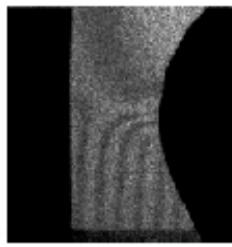

$403 \mathrm{~V}$

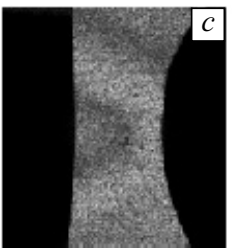

$2422 \mathrm{H}$

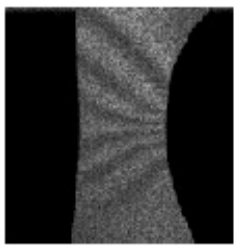

$2423 \mathrm{~V}$

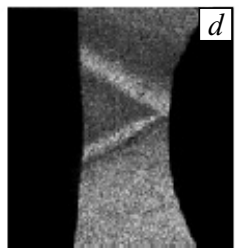

$2741 \mathrm{H}$

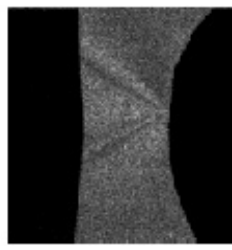

2742 V

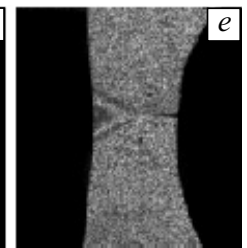

$3175 \mathrm{H}$

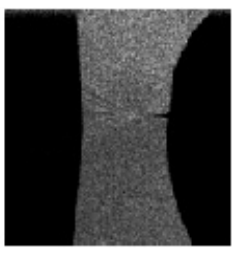

$3176 \mathrm{~V}$

Fig. 2. Horizontally (top row) and vertically (bottom row) sensitive fringes

electronic speckle-pattern interferometry on the front side is sensitive to horizontal displacement of the specimen and the one on the rear side is sensitive to vertical displacement. Mirrors are arranged to image both sides of the specimen on the same charge-coupled device camera so that the specklegrams associated with the horizontal displacement and vertical displacement can be captured simultaneously. The specimen is a tin plate of $20 \mathrm{~mm}$ wide, $100 \mathrm{~mm}$ long, and $0.4 \mathrm{~mm}$ thick. One side of the specimen is curved so that the width of the vertical center is narrowest $(15 \mathrm{~mm})$ and thereby fracture is always initiated on this side at the vertical center (Fig. 2). The specklegrams are taken at $30 \mathrm{frames} / \mathrm{s}$ as the tensile machine applies the load at a constant pulling rate of $4 \mu \mathrm{m} / \mathrm{s}$.

By subtracting the specklegram taken at a certain time step from the specklegram taken at another time step, a differential specklegram, called the interferogram, can be obtained. Each interferogram has an intensity profile corresponding to the difference of Eqs. (8) and (9). Figure 2, as an example, shows interferograms obtained at several time steps in the same tensile experiment. As these images indicate, each interferogram shows a number of dark fringes. Each dark fringe represents the contour of displacement whose phase change ( $\phi$ in Eq. (9)) is an even integer multiple of $\pi$.

\section{Interpretation of fringe patterns}

By approximating the dark fringes observed in Fig. 2 with second order polynomials, we can express the corresponding displacement as follows:

$$
\begin{aligned}
& u(x, y)=a_{2} x^{2}+a_{1} x+b_{2} y^{2}+b_{1} y=m u_{0}, \\
& v(x, y)=c_{2} x^{2}+c_{1} x+d_{2} y^{2}+d_{1} y=n v_{0},
\end{aligned}
$$

where $u$ and $v$ denote the horizontal and vertical displacement, respectively, $m$ and $n$ are integers, and $u_{0}$ and $v_{0}$ represent the unit displacement corresponding to the phase change of $\phi=2 \pi$. It is reasonable to eliminate the term proportional to $x y$ because the curved fringes are symmet- ric about the $x$ or $y$ axis (Fig. 2). With these polynomial approximations, different stages of deformation can be expressed as follows.

\subsection{Elastic condition}

The elasticity is characterized as coordinate independent distortion tensor components, i.e., the first order derivatives of displacement $(\partial u / \partial x, \partial u / \partial y$, etc.) are constant. In Eqs. (10) and (11), this means that the coefficients for the second order terms are all zero, or the dark fringes are equally-spaced straight lines. If this condition is true, the deformation is elastic.

\subsection{Plastic condition}

If the above condition of elasticity is not true, the deformation is plastic. It follows that if the dark fringes are curved or not equally spaced, the deformation is plastic.

\subsection{Critical fracture condition}

In the $x y$ plane, the critical fracture criterion (6) and (7) can be expressed as follows:

$$
\begin{aligned}
(\nabla \times \boldsymbol{\omega})_{x} & =\frac{\partial \omega_{z}}{\partial y}=\frac{\partial^{2} v}{\partial x \partial y}-\frac{\partial^{2} u}{\partial y^{2}}= \\
& =-2 b_{2} y=0, \text { i.e., } b_{2}=0, \\
(\nabla \times \boldsymbol{\omega})_{y} & =-\frac{\partial \omega_{z}}{\partial x}=-\left(\frac{\partial^{2} v}{\partial x^{2}}-\frac{\partial^{2} u}{\partial x \partial y}\right)= \\
& =-2 c_{2} x=0, \text { i.e., } c_{2}=0,
\end{aligned}
$$

Possible combinations of coefficient of $u(x, y)$ to satisfy condition (12)

\begin{tabular}{c|c|c|c|c|c|c|c|c}
\hline Case & 1 & 2 & 3 & 4 & 5 & 6 & 7 & 8 \\
\hline$a_{2}$ & $\neq 0$ & $\neq 0$ & $\neq 0$ & $\neq 0$ & $=0$ & $=0$ & $=0$ & $=0$ \\
\hline$a_{1}$ & $\neq 0$ & $\neq 0$ & $=0$ & $=0$ & $\neq 0$ & $=0$ & $\neq 0$ & $=0$ \\
\hline$b_{1}$ & $\neq 0$ & $=0$ & $\neq 0$ & $=0$ & $=0$ & $\neq 0$ & $\neq 0$ & $=0$ \\
\hline
\end{tabular}


Equation and shape of horizontally sensitive fringes $u(x, y)$ under critical fracture condition

\begin{tabular}{c|c}
\hline Case & Equation and shape of fringes \\
\hline 1 & $a_{2} x^{2}+a_{1} x+b_{1} y=m u_{0}$, vertical parabolas \\
\hline 2 & $a_{2} x^{2}+a_{1} x=m u_{0}$, compressed, vertical straight lines \\
\hline 3 & $a_{2} x^{2}+b_{1} y=m u_{0}$, vertical parabolas \\
\hline 4 & $a_{2} x^{2}=m u_{0}$, compressed, vertical straight lines \\
\hline 5 & $a_{1} x=m u_{0}$, equally-spaced, vertical straight lines \\
\hline 6 & $b_{1} y=m u_{0}$, equally-spaced, horizontal straight lines \\
\hline 7 & $a_{1} x+b_{1} y=m u_{0}$, equally-spaced, slant straight lines \\
\hline 8 & trivial \\
\hline
\end{tabular}

Table 3

Equation and shape of vertically sensitive fringes $v(x, y)$ under critical fracture condition

\begin{tabular}{c|c}
\hline Case & Equation and shape of fringes \\
\hline $1^{\prime}$ & $d_{2} y^{2}+d_{1} y+c_{1} x=n v_{0}$, horizontal parabolas \\
\hline $2^{\prime}$ & $d_{2} y^{2}+d_{1} y=n v_{0}$, compressed, horizontal straight lines \\
\hline $3^{\prime}$ & $d_{2} y^{2}+c_{1} x=n v_{0}$, horizontal parabolas \\
\hline $4^{\prime}$ & $d_{2} y^{2}=n v_{0}$, compressed, horizontal straight lines \\
\hline $5^{\prime}$ & $d_{1} y=n v_{0}$, equally-spaced, horizontal straight lines \\
\hline $6^{\prime}$ & $c_{1} x=n v_{0}$, equally-spaced, vertical straight lines \\
\hline $7^{\prime}$ & $d_{1} y+c_{1} x=n v_{0}$, equally-spaced, slant straight lines \\
\hline $8^{\prime}$ & trivial \\
\hline
\end{tabular}

$$
\begin{aligned}
\mathbf{j} & =\varepsilon\left(\frac{\partial u}{\partial x}+\frac{\partial v}{\partial y}\right) \mathbf{W}_{\mathrm{d}}= \\
& =\varepsilon\left(2 a_{2} x+a_{1}+2 d_{2} y+d_{1}\right) \mathbf{W}_{\mathrm{d}} \neq 0,
\end{aligned}
$$

where Eq. (4) is used in Eq. (14). Equation (12) indicates that under the critical fracture condition the horizontally sensitive fringes $u(x, y)$ does not have the second order dependence on $y$ while it can have the second order dependence on $x$. Similarly, Eq. (13) indicates that the vertically sensitive fringes $v(x, y)$ does not have the second order dependence on $x$ but can have the second order dependence on $y$. Table 1 lists all the possible combinations for the co-

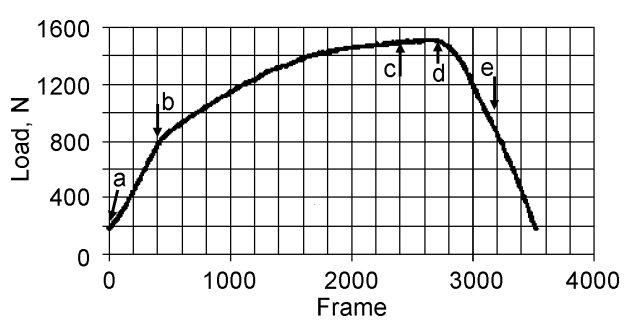

Fig. 3. Loading characteristics of specimen shown in Fig. 2

efficients of the horizontally sensitive fringes to satisfy condition Eq. (12), and Table 2 shows the shape and expression of the dark fringes for each combination. Table 3 shows the corresponding cases for the vertically sensitive fringes.

The fringe shapes observed in Tables 2 and 3 can be conveniently summarized as follows. If horizontally/vertically sensitive fringes lose their horizontal/vertical curvature, it means that the critical fracture condition is satisfied. Equivalently, if horizontally/vertically sensitive fringes show a horizontal/vertical curvature, the material is in the plastic regime but does not meet the critical fracture condition.

With these in mind, we now analyze the fringe patterns seen in Fig. 2. The images in the upper and lower rows are, respectively, the horizontally and vertically sensitive fringes. The pair of the images in the same column are the fringes formed from the specklegrams taken at the same time steps. Figure 3 is the loading characteristics of the specimen shown in Fig. 2. The labels $a-e$ in Fig. 3 indicate the points on the loading curve when the fringe patterns $a-e$ in Fig. 2 are formed. The following observations support the above argument regarding the relationships between the fringe shapes and the stage of deformation.

Fringes in Fig. 2(a) represent elastic deformation. Fringes are all straight lines and equally spaced. Figure 3 supports this observation indicating that the stress-strain relationship is in the linear range when these fringes are formed.

Fringes in Fig. 2(b) correspond to the point where the linear stress-strain relationship is about to finish (Fig. 3).
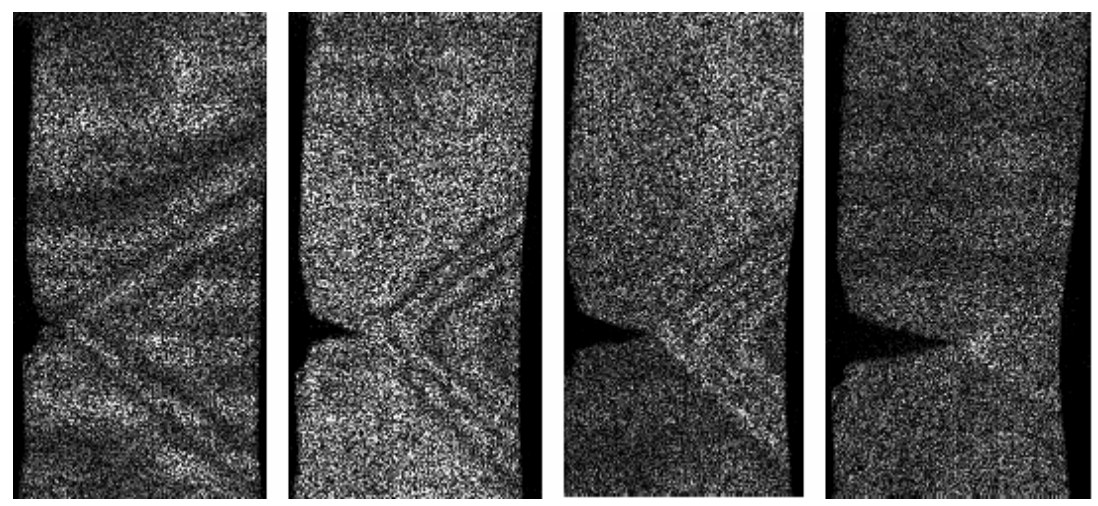

Fig. 4. Fringe pattern moving with a crack tip 

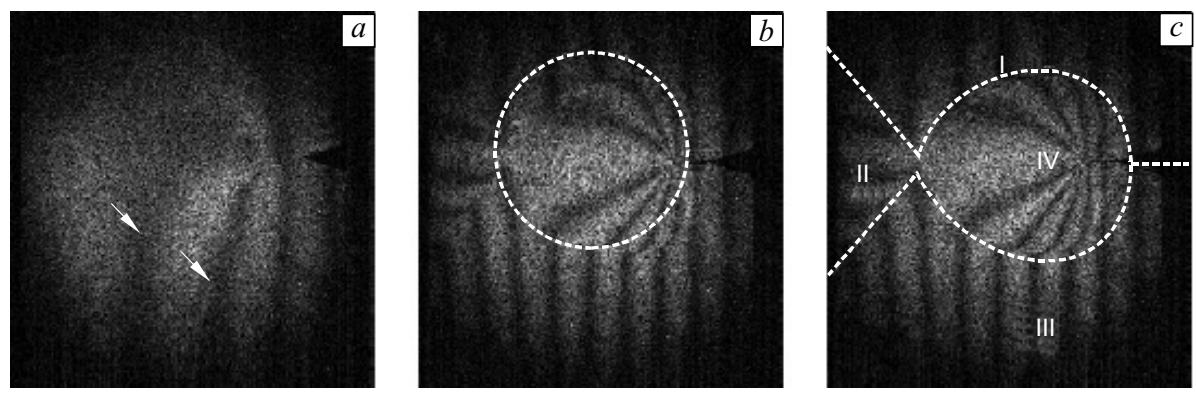

Fig. 5. Vertically sensitive fringes observed in a thicker steel specimen $(a)$, the specimen deforms plastically in the entire view $(b)$, the near crack-tip region is divided into elastic and plastic zones, as indicated in $(c)$. The time elapsed from a reference time is $3.8(a), 10.2 \mathrm{~s}(b$ and $c)$

Note that near the vertical center of the images, the horizontally/vertically sensitive fringes show horizontal/vertical parabolic shapes. In accordance with the above argument, this indicates that the material deforms plastically but has not reached the critical fracturing stage. The same tendency (the horizontally/vertically sensitive fringes show horizontal/vertical parabolas) continues till point $c$ in Fig. 3 .

At point $d$ in Fig. 3, the horizontally/vertically sensitive fringes lose the horizontal/vertical curvatures, becoming straight lines. These satisfy the critical fracture conditions 7 and $7^{\prime}$. Indeed, short after this point, the loading curve begins to decrease supporting this observation. It is interesting to note that the critical fracture condition is satisfied in the horizontally and vertically sensitive fringes at the same time.

Fringes in Fig. 2(e) are typically observed in the last stage of deformation where the load decreases monotonically. Normally, the horizontally and vertically sensitive fringes show similar slant straight lines that move with the crack tip until the specimen completely fractures. Figure 4 shows an example of such a pattern observed in horizontally sensitive fringes obtained in a different experiment. The fringe pattern can be classified as case 7 of Table 2 . Apparently, $\partial u / \partial x \neq 0$, and condition (14) is satisfied.

Figure 5 shows vertically sensitive fringes observed in a similar tensile experiment in which a carbon steel (S50C) specimen thicker $(62 \mathrm{~mm}$ wide, $70 \mathrm{~mm}$ long, and $13 \mathrm{~mm}$ thick) than the tin specimen is used at a higher pulling rate $(100 \mu \mathrm{m} / \mathrm{s})$. At $t=3.8 \mathrm{~s}$, two vertically parabolic fringes (highlighted by the arrows) are observed. However, at $t=$ $=10.2 \mathrm{~s}$, the fringes are horizontally parabolic (inside the circle) or straight. In Fig. $5(\mathrm{c})$, the fringe pattern at $t=10.2 \mathrm{~s}$ is divided into four zones based on the shapes of the fringes. In zones I and III, the fringes are equally-spaced, vertical and straight; in zone II, the fringes are equally-spaced, horizontal and straight, and in zone IV the fringes are horizontally parabolic. According to the criteria shown in Table 3 , these observations can be explained as follows. As of $3.8 \mathrm{~s}$, the specimen deforms plastically in the entire view of the fringe pattern. At a certain point of time within the next $6.4 \mathrm{~s}$, the near crack-tip region is divided into elastic zones (zones I-III) and the plastic zone (zone IV). Judging from the orientations of the straight fringes, it is likely that in zones I and III the specimen is experiencing counterclockwise and clockwise rotations, respectively, and in zone II it is experiencing compression due to the two opposite rotations. Moreover, as of $10.2 \mathrm{~s}$, the fringes in the plastic zone satisfy the critical fracture criterion. Indeed, the specimen fractured at $10.3 \mathrm{~s}$.

In summary, a method to visualize the plastic zone in the near crack-tip displacement field has been proposed and demonstrated. Using the plastic and critical fracture criteria derived from physical mesomechanics, this method is capable of diagnosing the visualized deformation as to whether the crack is about to open. Considering that these criteria are based on a fundamental physical principle, this method is expected to be applicable to materials showing ductile fracture in general.

\section{Acknowledgments}

The authors are grateful to J. Gerike of Southeastern Louisiana University for his technical support and the Southeastern University Alumni Association for their financial support. Discussion with T. Sasaki of Southeastern Louisiana University is also appreciated.

\section{References}

[1] A.S. Tetelman and A.J. McEvily, Jr., Fracture of Structural Materials, John Wiley \& Sons, Inc., New York, 1967.

[2] Speckle Metrology, Ed. by R.S. Sirohi, Marcel Dekker, Inc., New York, 1993.

[3] Physical Mesomechanics of Heterogeneous Media and ComputerAided Design of Materials, Ed. by V.E. Panin, Cambridge Interscience Publishing, Cambridge, 1998.

[4] S. Yoshida, Physical mesomechanics as a field theory, Phys. Mesomech., 8, No. 5-6 (2005) 15.

[5] L.D. Landau and E.M. Lifshitz, Theory of Elasticity. Vol. 7. Course of Theoretical Physics, Butterworth-Heinemann, Oxford, 1986.

[6] V.E. Egorushkin, Dynamics of plastic deformation: Waves of localized plastic deformation in solids, Russ. Phys. J., 35, No. 4 (1992) 316.

[7] S. Yoshida, B. Siahaan, M.H. Pardede, N. Sijabat, H. Simangunsong, T. Simbolon and A. Kusnowo, Observation of plastic deformation wave in a tensile-loaded aluminum-alloy, Phys. Lett. A, 251, No. 1 (1999) 54.

[8] S. Yoshida, Dynamics of plastic deformation based on restoring and energy dissipative mechanisms in plasticity, Phys. Mesomech., 11, No. 3-4 (2008) 137.

[9] S. Yoshida, Consideration on fracture of solid-state materials, Phys. Lett. A, 270, No. 6 (2000) 320. 